\title{
INJECTOR FOR RFQ USING ELECTROSTATICALLY FOCUSED TRANSPORT AND MATCHING
}

\author{
O.A. Anderson, L. Soroka, J.W. Kwan, and R.P. Wells* \\ Accelerator and Fusion Research Division \\ Lawrence Berkeley Laboratory \\ University of California \\ 1 Cyclotron Road, Berkeley, CA 94720
}

\begin{abstract}
We discuss the principles and performance of a new type of high-current $\mathrm{H}^{-}$ injector for RFQs. The distinguishing feature of our injector is that we replace the conventional gas-neutralized transport and matching units by electrostatic focusing units. Our system prevents plasma formation along the beam instead of utilizing it. Some advantages of this approach are discussed.
\end{abstract}

\section{INTRODUCTION}

This paper describes the design and preliminary operation $\mathrm{c}^{\text {? }}$ a de $\mathrm{H}^{-}$injector for a radio frequency quadrupole (RFQ). The system tested consists of a volume producrion ion source [1], a 100-keV electrostatic preaccelerator [2], and a new electrostatic transport and matching system. The injector has been tested with up to $45 \mathrm{~mA}$ of $\mathrm{H}^{-}$, but simulations show that it is capable of handling beams with several times higher current. To allow for dc operation, all components are water cooled.

As discussed previously [2], the $\mathrm{H}^{-}$volume source operates at a pressure of more than $10 \mathrm{~m}$ Torr. To minimize stripping of the $\mathrm{H}^{-}$beam, we use an open accelerator and transport structure, with high conductance to pumps. Electrons from the source are removed by an electron trap mounted on the extractor grid [2].

We use electrostatic quadrupole (ESQ) focusing for our low energy beam transport (LEBT) and an axisymmetric lens for matching into the RFQ. The ESQ LEBT, described in Section 2, provides sufficient length for effective pumping while prevent-

\footnotetext{
"This work was sujpu:ted ty USASDC Contract No. MIPR W31RPD-63-A087 and by the Director, Office of Energy Researct., Office of Fusion Energy, Development and Technology Division, of the U.S. Department of Energy under Contract No. DE-AC03-76SF00098.
} 
ing the accumulation of charge and the formation of plasma in the transport channel. The final match is obtained with an axisymmetric electrostatic lens rather than with ESQ focusing, as discussed in Section 3. Experimental results are given in Section 4.

\section{ELECTROSTATICQUADRUPOLE LEBT}

Our new approach for transport of intense $\mathrm{H}^{-}$beams uses ESQ focusing instead of the usual combination of magnets and gas neutralization [3,4]. Some of the fearures of our plasma-free LEBT are: (1) The open structure gives good gas pumping capability. (2) Gas pressure can be arbitrarily low in the LEBT; gas independence improves reproducibility. (3) Gas independence permits either dc operation or pulsed beams with arbirarily short pulse lengths. (4) Electrostatic tuning provides excellent flexibility. (5) Emittance growth from plasma noise is eliminated. (6) Also eliminated is emittance growth from sheath transitions into and out of gas neutralized regions. (7) The deneutralization transition near the match point in the $R F Q$, a tricky issue in conventional designs, is avoided.

Our LEBT uses ESQ technology [5] which was developed and tested for the DOE magnetic fusion energy program [6] and adapted for the present application.

\subsection{Field Strength Compared with Magneric (Gas Neumalized) Case}

\section{Electrostaric auadrupole focusing}

The quadrupole pole face electric field $E_{Q}$ required to transport a specified beam current I with normalized emittance $\epsilon_{\mathrm{N}}$ at beam energy $q \mathrm{q}$ is given by [7]

$$
\frac{L^{2}}{a_{O}^{2}} E_{0}^{2}=C_{1} \frac{I}{A_{0}^{2}} V^{1 / 2}+C_{2} \frac{E_{N}^{2}}{A_{0}^{4}} V
$$

for a matched bearn: $L$ is the quad cell length, $a_{Q}$ is the quad aperture radius, and $A_{0}$ is the mean beam radius. The constants $C_{1}$ and $C_{2}$ depend on the particle sharge and mass and the electrode cecupancy factor, $C_{2}$ also includes a correction for beam ripple which is usually negligible at higher energies where the $\epsilon_{N}$ term becomes significant [7].

The $\mathrm{E}_{\mathrm{Q}}{ }^{2}$ external force term on the left of Eq. (1) balances the space charge and emittance pressure terms on the right. In a typical LEBT, $E_{Q} \approx 10 \mathrm{kV} / \mathrm{cm}$. For a bright 
beam transpcitid at low energy, the emittance term is usually negligible, and

$$
E_{0} \propto V^{1 / 4}
$$

We routinely use Eq. (1) or (1') when designing ESQ accelerators and LEBTs.

\section{Magnetic gas neutralized focusing}

If the electrostaric quadrupoles are replaced by magnetic quadrupoles, then

$$
E_{0}^{2} \longrightarrow C_{3} \vee B_{0}^{2}
$$

For a $100 \mathrm{keV} \mathrm{D}$ beam, $10 \mathrm{kV} / \mathrm{cm}$ is approximately equivalent to $3 \mathrm{kG}$. However, in the absence of external electrostatic fields, a neutralizing plasma develops. We define the neurralization coefficient $\mathrm{K}_{\mathrm{n}}$, where typically $\mathrm{K}_{\mathrm{n}} \approx 0.99$, and get

$$
C_{3} \frac{L^{2}}{a_{O}^{2}} B_{Q}^{2}=C_{1}\left(1-K_{n}\right) \frac{I}{A_{0}^{2}} V^{-1 / 2}+C_{\frac{C_{N}}{2}}
$$

In Eq. (2), note that the factor $\left(1-K_{n}\right)$ is very sensitive to the degree of neutralization; for example, it doubles if $K_{n}$ changes from 0.99 to 0.98 . We also note that $K_{n}$ is a poorly known function of position and time in a magnetic LEBT, so that Eq. (2) is not useful for design purposes unless the emittance term is large enough to dominate. Thus, the design of magnetic LEBTs must be largely empirical.

\subsection{Mechanical Configuration of Experimental LEBT}

The electrostatic LEBT which we tested is shown in Fig. 1. The hardware is the same as used for the $200 \mathrm{kV}$ Constant Current Variable Voltage (CCVV) prototype accelerator tested and described previously $[5,8,9]$. Two ESQ modules containing a total of five sets of quadrupoles are shown in the figure. In the accelerator prototype application, the first module is for matching and the second is for acceleration. The initial quadrupole in the matching module has been shortened to facilitate the transition from an initially round beam to a beam matched for transport or acceleration. The second module is the first of an evenrual series of identical acceleration modules [9], 
and therefore does not have a shortened exit quadrupole.

Even though the hardware shown in Fig. 1 was not designed for LEBT applications, it proved to be perfectly adequate as a demonstration LEBT capable of handling parameters of interest in all known RFQ injector applications. Naturally, a dedicated electrostatic LEBT would be designed differently, with fewer elements and shorter overall length.

The CCVV accelerator that we adapted for our LEBT experiment was developed for applications that require constant beam current over a wide energy range. Flexibility in energy results from the use of transverse field ESQ focusing in the main accelerator rather than the use of longitudinal field focusing as in conventional Pierce columns. Therefore it is easy to reduce the output beam energy from the nominal $200 \mathrm{keV}$ CCVV level to the $100 \mathrm{keV}$ level used in the LEBT mode. In fact, much lower energies can be reached without loss of beam current, because the preaccelerator voltage, which determines the beam current, is always kept at the same value [5].

Operation in the LEBT mode requires not only reduction in the output energy as just mentioned, but also adjustment of the beam shape. In the CCVV accelerator mode, the exit beam is elliptical in cross section, whereas the RFQ LEBT needs to produce a round beam. This adjusument can also be made, as shown in the next section.

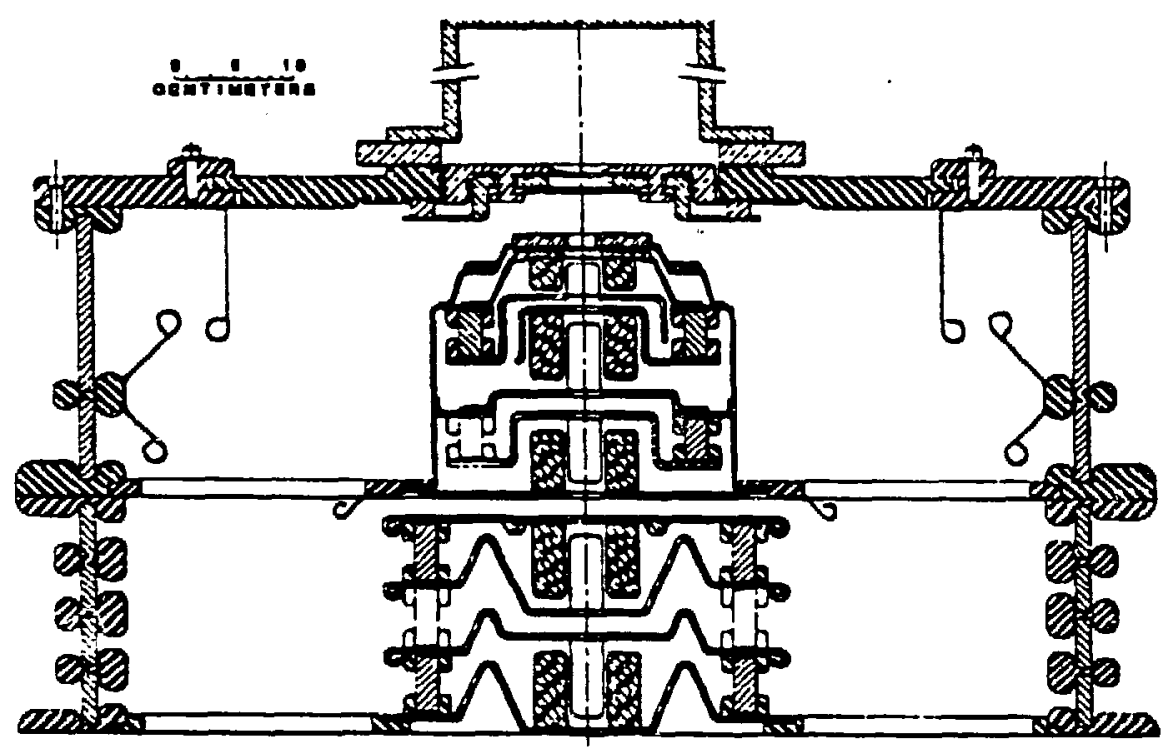

Fig. 1. ESQ system used for testing $100 \mathrm{keV}$ electrostatic LEBT principle. Running in its accelerator mode, this system is the $200 \mathrm{keV}$ prototype for a MeV dc accelerator with fusion energy applications. 


\section{2,3 Beam Simularions for Experimental LEBT}

Fig. 2 shows an envelope simulation for the hardware of Fig. 1 running in the LEBT mode. Corresponding to a particular experimental run, the preaccelerator was assumed to inject $45 \mathrm{~mA}$ of $\mathrm{H}^{-}$in a round beam of $0.8 \mathrm{~cm}$ radius into the LEBT. The normalized emittance is $0.160 \pi \mathrm{mrad}-\mathrm{cm}$. The ESQ voltages were adjusted to produce a round beam at the exit with a diverging envelope (about $24 \mathrm{mrad}$ ) as required for the aperture lens module described in the next section. The voltage variation across the beam is small because no attempt is made to use the ESQ voltages for matching into the RFQ. That function is reserved for the aperture-lens module discussed in Section 3.

We have also recently started running self consistent 3-D particle simulations for the ESQ structure of Fig. 1, using a version of the ARGUS code [10] especially developed for our purposes by SAIC [11]. Fig. 3 shows preliminary results from a test run by SAIC for the acceleration mode. In the near future we will be able to do 3-D LEBT mode simularions at LBL which will allow exact predictions for emitrance growth.

\section{AXISYMMETRIC APERTURE-LENS MATCHING MODULE}

A difficult problem in designing RFQ injectors is in the beam matching: a small beam diameter and large convergence angle are required at the match point just inside the RFQ entrance. We found that producing a round beam of this sort, using only ESQ

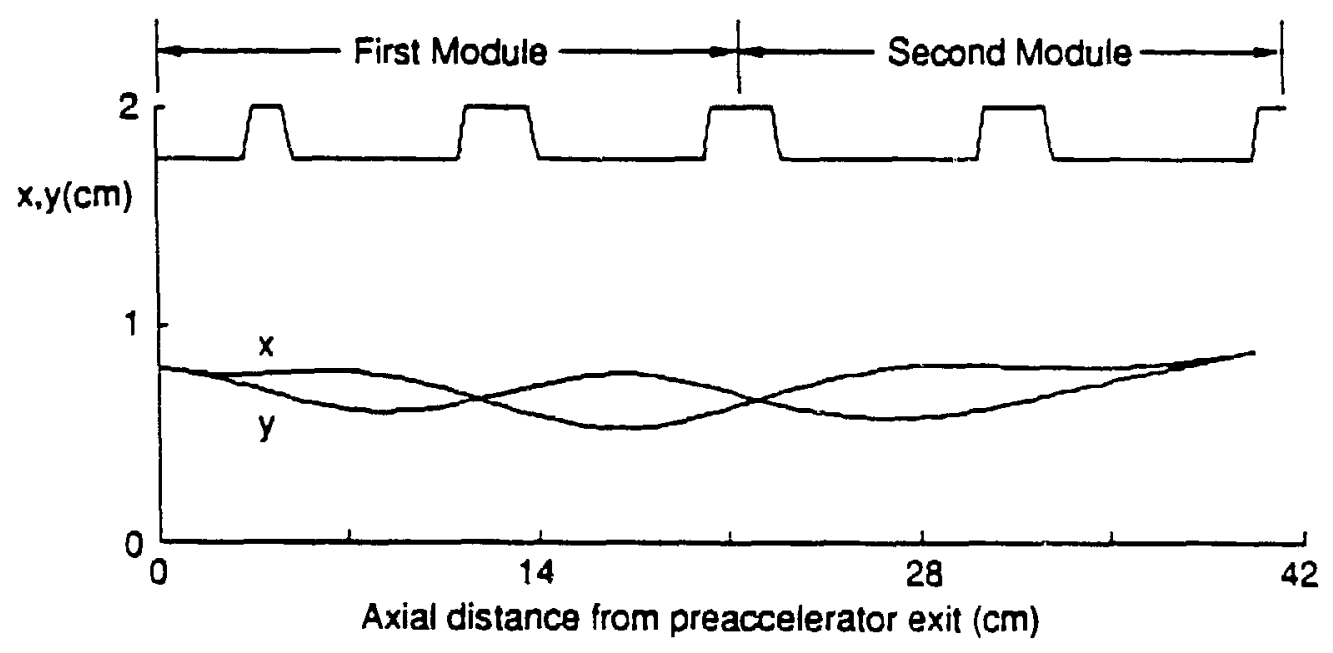

Fig. 2. Beam envelope simulation for the system of Fig. 1, running in LEBT mode. Parameters are given in the text. 
units, requires relatively large focusing voltages which could produce excessive aberrations. Since low emittance growth is one of our goals, we chose the alternative of axisymmetric focusing between the LEBT and RFQ. Although axisymmetry per se does not guarantee improvement, a big advantage is that the design can be optimized with exact 2-D round-beam particle codes, which run much faster than 3-D particle codes. We used the self-consistent 2-D WOLF code [12] to model our matching module.
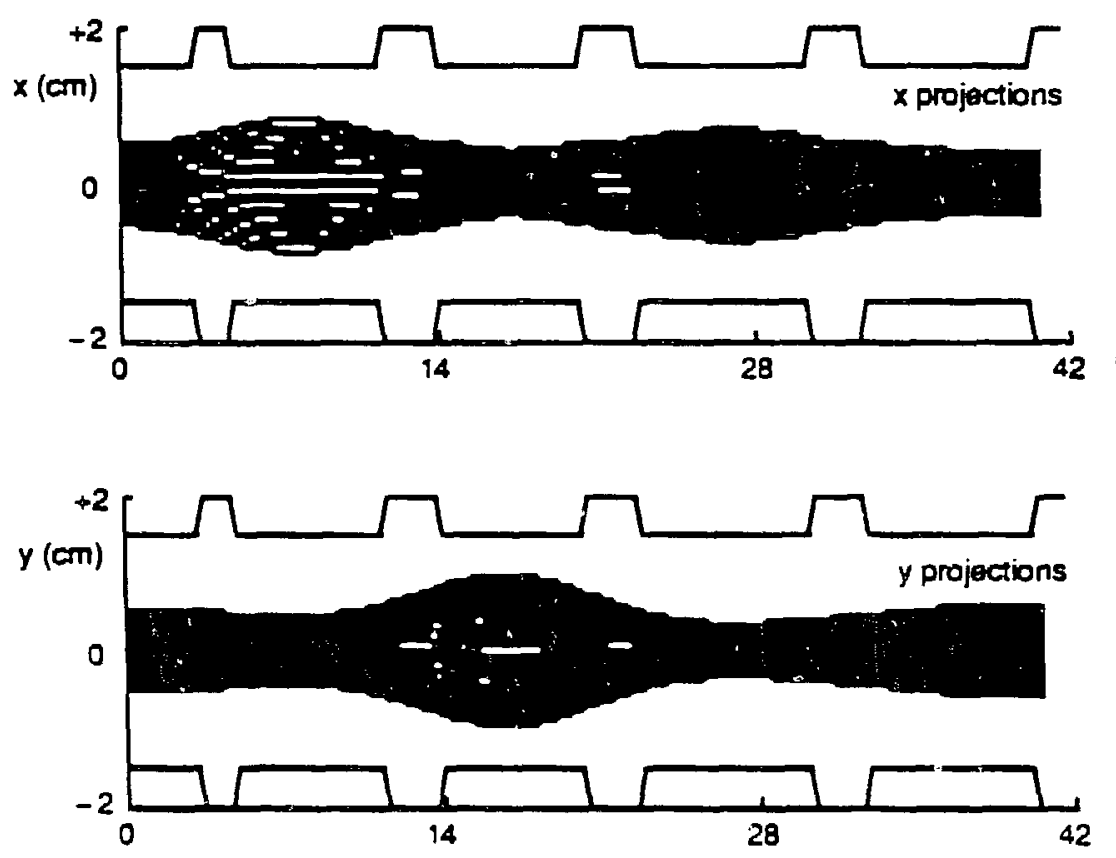

Fig. 3. Beam trajectories for the device of Fig. 1, running in accelerator mode with $200 \mathrm{~mA}$ of $\mathrm{H}^{-}$. The trajectories are shown projected in the $x$ and $y$ directions.

\section{Consmuction of Ring Lens}

Fig. 4 shows a third module added to the two ESQ modules of Fig. 1; this module is our axisymmetric lens. One sees that it is nor a conventional einzel lens; by definition, einzel lenses have field-free drift regions, which we wish to avoid, since plasma can accumulate in such regions. On the other hand, we do not use a classical thin-plate aperture lens because we wish to avoid electric field concentracions. Our compromise design looks like a ring (near the beam), and we simply call it a ring lens.

The figure shows one of three insulators which support the ring. These insulators 
also serve as vacuum feed-throughs, providing both electrical current and cooling water for the ring lens electrode. This electrode is a two piece assembly with the outer ring serving as a support for an inner ring which can be shimmed and translated to accurately align the electrode bore with the beam axis. Not shown is a movable, grounded, exit electrode containing a small aperture simulating the entrance of the RFQ. The exit electrode is positioned by a remotely controlled actuator that replaces the small aperture by a large one during tune up of the ESQ portion of the LEBT.

Depending on requirements, a voltage in the range of -95 to $-98 \mathrm{kV}$ (with respect to ground) is applied to the ring during operation. Note that the ring is actually energized by a small $2-5 \mathrm{kV}$ floating power supply connected to the $-100 \mathrm{kV} \mathrm{H}^{-}$ion source potential.

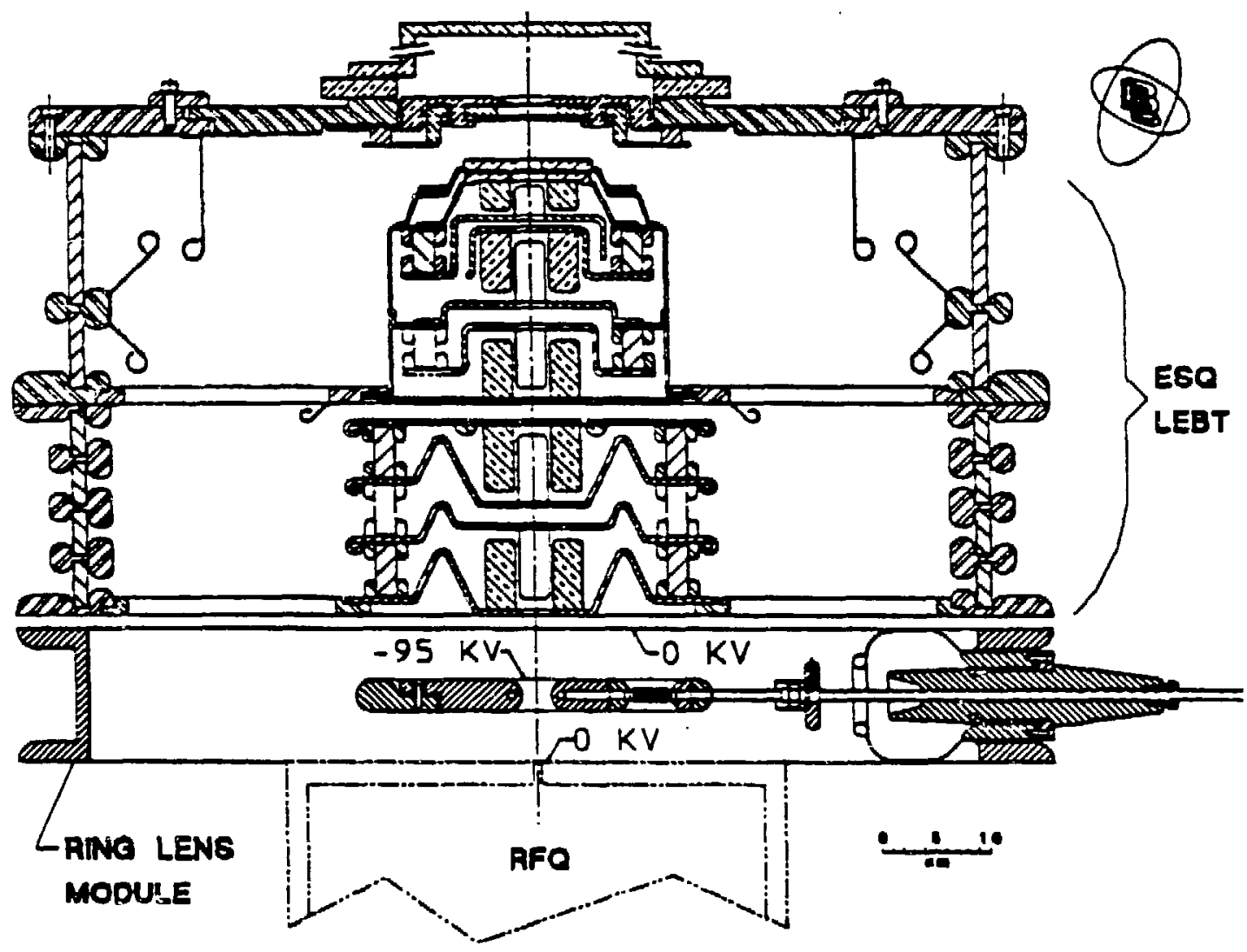

Fig. 4. LEBT shown with ring lens added. As described in the text, the ring is supported by tinree feed through insulators, one of which is seen here. The hypothetical RFQ entrance region shown schematically with dotted lines is replaced experimentally by the exit electrode described in the text. 


\section{Self Consistent Particle Simulation of Ring Lens}

Fig. 5 shows a sample particle simulation for the ring lens system of Fig. 4 , cbtained with the self-consistent WOLF particle code [12]. Parameters are given in the figure; the current is larger than for Fig. 2, so this case is more stringent. The equipotentials show that the beam is decelerated from $100 \mathrm{keV}$ to about $15 \mathrm{keV}$ and reaccelerated to $100 \mathrm{keV}$ at the RFQ input. Aberrations are small, and the emittance growth is only $2.9 \%$.

It would be interesting to compare the aberrations for the ring lens and the ESQ focusing options for RFQ entrance matching. This will be possible in the near future using the ARGUS code $[10,11]$ for the ESQ option.

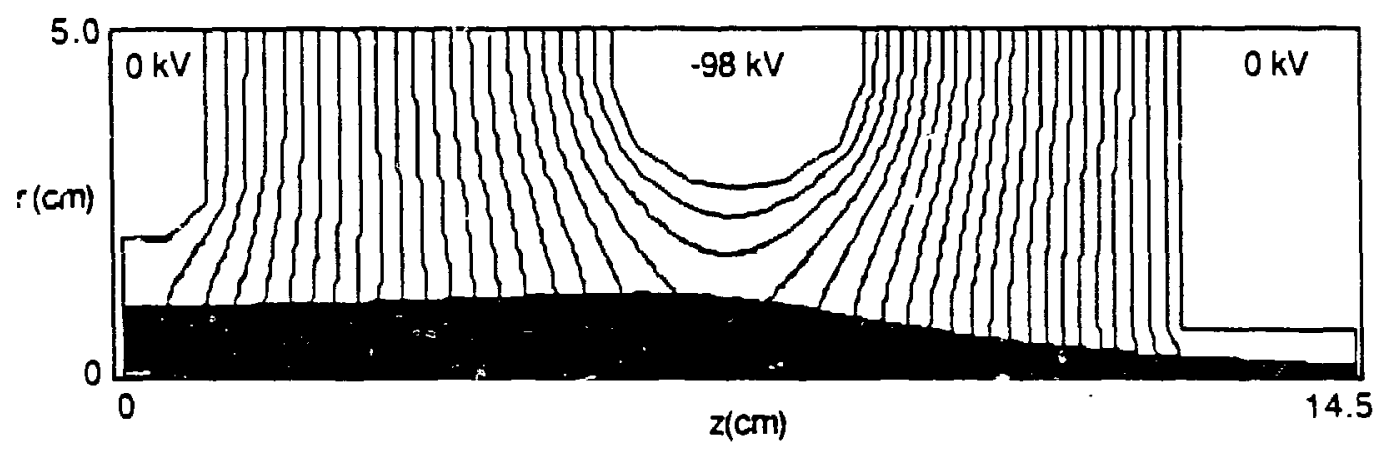

\begin{tabular}{|ll|}
\hline Particle & $\mathrm{H}^{-}$ \\
Energy (keV) & 100 \\
Current (mA) & 100 \\
$\epsilon_{\mathrm{N}}(\pi \mathrm{cm}$-mrad) & 0.014 \\
Entrance radius (cm) & 1.00 \\
Entrance angle (mrad) & 0 \\
Exit radius (cin) & 0.299 \\
Exit angle (mrad) & -71 \\
\hline
\end{tabular}

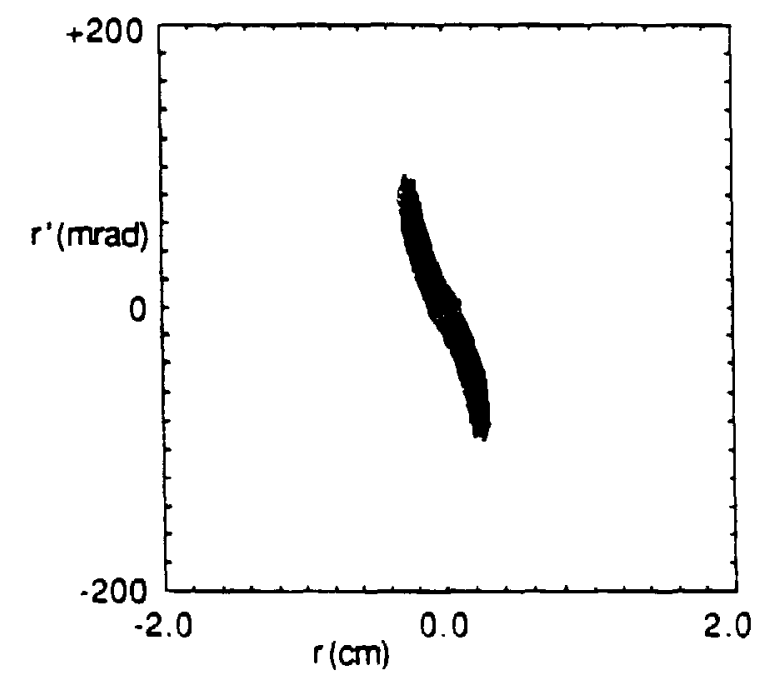

Fig. 5. Sample particle simulation of the axisymmetric lens for RFQ matching, showing the beamlet trajectories and the phase plot at $12.6 \mathrm{~cm}$, the location of the simulated RFQ entrance. The beam radius is $3 \mathrm{~mm}$ at this point and decreases toward the RFQ match poin:. 


\section{EXPERIMENTAL RESULTS}

Preliminary test results are shown in Fig. 6. These were obtained using our large aperture $\mathrm{H}^{-}$volume source [13]. For the run shown, the extraction grid diameter was reduced to $1.4 \mathrm{~cm}$ in order to improve the current density. In this particular run, the source produced a rather asymmetric beam with a lump on one edge, as seen in Fig. 6 a.

(a)

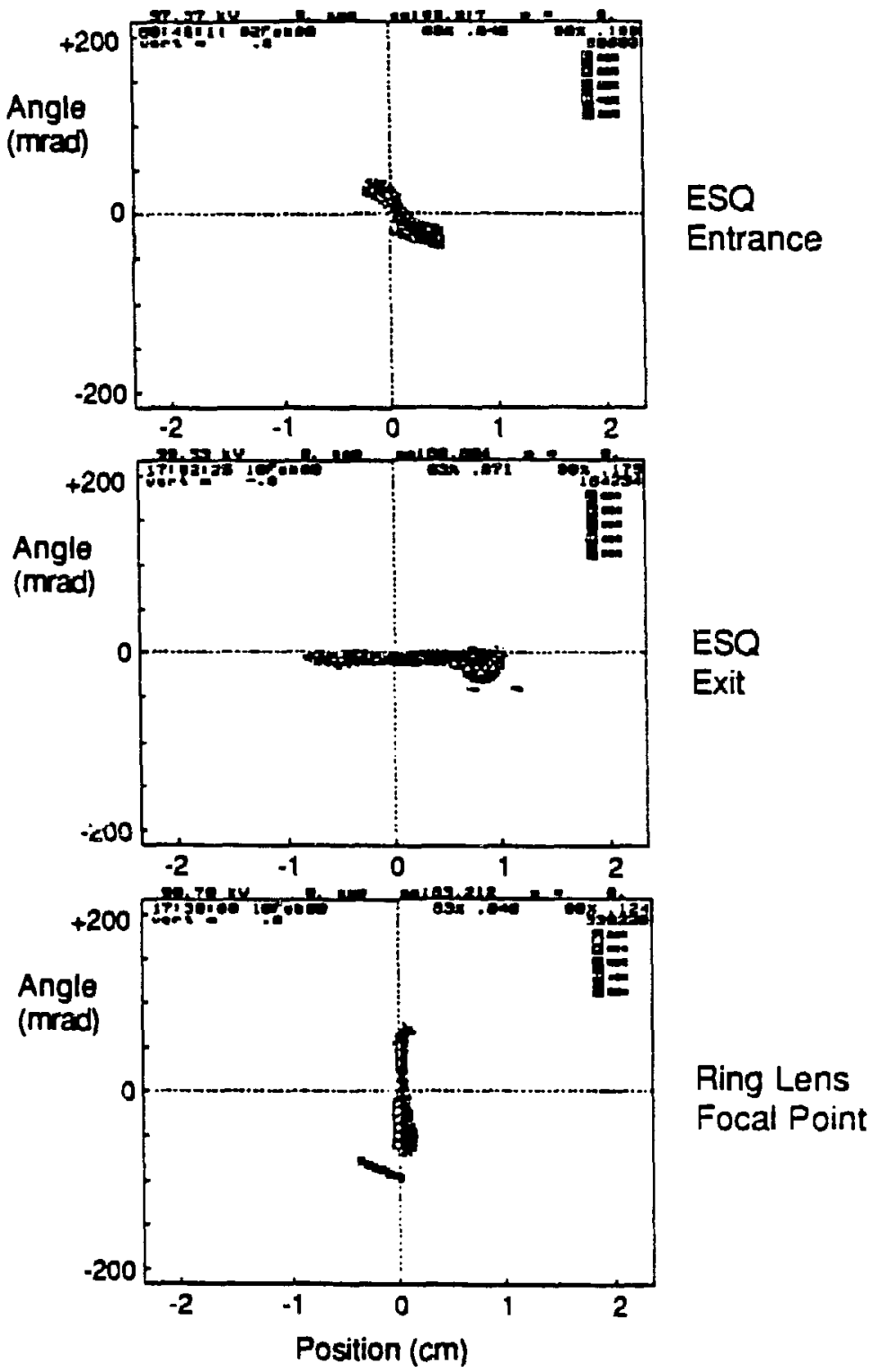

Fig. 6. Emittance scans projected back to: (a) the ESQ entrance; (b) the ESQ exit; and (c) the ring lens focal point. The larger phase space area seen in (b) is probably an instrumental effect. In (a) and (c) the normalized emittance is about $0.05 \pi \mathrm{mrad}-\mathrm{cm}$. 
The lump grows as it is transported through the LEBT (Fig. 6b) and appears to be somewhat disconnected at the ring lens focus (Fig. $6 c$ ). The important point, however, is that the bulk of the phase plots are essentially straight and free of aberration after passing through the LEBT and after passing through the ring lens.

In this run, the LEBT operated at $100 \mathrm{kV}$. The transported $\mathrm{H}^{-}$current was 30 $\mathrm{mA}$, primarily limited by the plasma generator. The beam loss, if any, was too small to measure. The beam exiring the LEBT at $10 \mathrm{~mm}$ radius was focused by the ring lens to a radius of $1 \mathrm{~mm}$; the convergence angle was $70 \mathrm{mrad}$ at the focal point.

By adjusting the ring voltage and the electrode spacings on each side of the ring, the matching module can handle a wide range of beam currents and can produce a wide range of convergence angles, up to $100 \mathrm{mrad}$.

\section{ACKNOWLEDGMENTS}

We would like to thank all the members of the MFE and HIFAR groups of the Accelerator and Research Division at LBL who contributed to this work. We are especially grateful to C.H. Kim (LBL), who gave us his variable-energy ESQ envelope code and helped us to use it.

\section{REFERENCES}

[1] K.N. Leung, K.W. Ehlers, and R.V. Pyle, "Optimization of $\mathrm{H}^{-}$Production in a Magnetically Filtered Multicusp Source," Rev. Sci. Instrum. 56, 364 (1985).

[2] O.A. Anderson, C.F. Chan, W.S. Cooper, W.B. Kunkel, J.W. Kwan, A.F. Lietzke, C.A. Matuk, P. Purgalis, and L. Soroka, "Design of a $200 \mathrm{~mA} \mathrm{DC} \mathrm{H} \mathrm{H}^{-}$Injector for an RFQ," Proc. of the 1987 Particle Accelerator Conference, Washington, DC, March 16-19, 1987; IEEE Cat. No. 87CH2387-9, p. 289.

[3] P. Allison and J.D. Sherman, "Operating Experience with a 100-keV, 100-mA H" Injector," Proc. 3rd Int'l Symposium on the Production and Neurralization of Negative Ions and Beams, Brookhaven National Laboratory, 1983; K. Prelec, Ed.; AIP Conf. Proc. 111, 511 (1983).

[4] J.G. Alessi, et al., "AGS Preinjector Improvement," Proc. of the 1987 Particle Accelerator Coníerence, Washington, DC, March 16-19, 1987; IEEE Cat. No. 87CH2387-9, p. 276. 
[5] O.A. Anderson, L. Soroka, C.H. Kim, R.P. Wells, C.A. Matuk, P. Purgalis, W.S. Cooper, and W.B. Kunkel, Proc. of the First European Particle Accelerator Conference, Rome, June 7-11, 1988; World Scientific Pub. Co, Singapore, p. 470 (1989).

[6] O.A. Anderson, et al., "A High Energy Neutral Beam System for Reactors," 15th Symposium On Fusion Technology, Utrecht, Netherlands, Sept. 19-23 1988; Elsevier Science Publishers B.V., p. 573 (1989).

[7] O.A. Anderson, "Integration Method for Alternating-gradient Problems with Space Charge," Lawrence Berkeley Laboratory report LBL-26123, to be submitted to Particle Accelerators.

[8] O.A: Anderson, L. Soroka, C.H. Kim, R.P. Wells, C.A. Matuk, P. Purgalis, J.W. Kwan, M.C. Vella, W.S. Cooper, and W.B. Kunkel, "Applications of the Constant-Current Variable-Voltage DC Accelerator," Nucl. Instrum. and Meth. B40I 41, 877 (1989).

[9] O.A. Anderson, W.S. Cooper, W.B. Kunkel, J.W. Kwan, R.P. Wells, C.A. Matuk, P. Purgalis, L. Soroka, M.C. Vella, G.J. De Vries, and L.L. Reginato, "The CCVV High-Current Megavolt Range DC Accelerator," 1989 Particle Accelerator Conf., Chicago, March 20-23, 1989; to be published by IEEE.

[10] A. Mankofsky, "Three-Dimensional Electromagnetic Particle Codes and Applications to Accelerators," Linear Acceleraror and Beam Opriss Codes, C.R. Eminheizer, ed., A.I.P. Conf. Proc. No. 177, 1988, p. 137.

[1:] A. Mondelli, C. Chang, A. Drobot, K. Ko, A. Mankofsky, and J. Petillo, "Application of the Argus Code to Accelerator Design Calculations," 1989 Particle Accel. Conf., Chicago, March 20-23, 1989; to be published by IEEE.

[12] W.S. Cooper, K. Halbach, and S.B. Magyary, "Computer-Aided Extractor Design," Proc. 2nd Siymp. cn Ion Sources and Formation of Ion Beams, Berkeley, CA; Lawrence Berkeley Laboratory report LBL-3399, p. II-1-6 (1974).

[13] J.W. Kwan, G.D. Ackerman, O.A. Anderson, C.F. Chan, W.S. Cooper, G.J. De Vries, K.N. Leung, A.F. Lietzke and W.F. Steele, "Operation of a DC Large Aperture Volume-Production Source," International Conf. on Ion Sources, Berkeley, CA, July 10-14, 1989; to be published by Rev. Sci. Instrum. 


\section{DISCLAIMER}

This document was prepared as an account of work sponsored by the United States Government. Neither the United States , Government nor any agency thereof, nor The Regents of the - University of California, nor any of their employees, makes any warranty, express or implied, or assumes any legal liability or ' responsibility for the accuracy, completeness, or usefulness of ' I any information, apparatus, pr duct, or process disclosed, or ' represents that its use would $\mathrm{r} \mathrm{\jmath}$ infringe privately owned rights. 'Reference 4rerein to any specific commercial products process, or , service by its trade name, trademark, manufacturer, or otherwise, does not necessarily constitute or imply its endorsement, 'recommendation, or favoring by the United States Government. or any agency thereof, or The Regents of the University of California. The views and opinions of authors expressed herein do not necessarily state or reflect those of the United States Government or any agency thereof or The Regents of the University of California and shall not be used for advertising or product endorsement purposes.

Lawrence Berkeley Laboratory is an equal opportunity employer. 